\title{
Effects of cross-modal transfer on direct and reversal learning in the rat
}

\author{
EUGENE R. DELAY \\ Regis College, Denver, Colorado
}

\begin{abstract}
In the first experiment, using within- and between-sensory modality CSs, direct and reversal transfer of active avoidance learning was studied in 48 male and 48 female albino rats. Training in each of two sessions employed either a high- or low-intensity light or noise CS. The analyses of both raw score data and proportional or savings score data indicated significant cross-modal transfer in direct as well as reversal learning conditions. Although females learned faster than males, there were no sex differences in amount of transfer. A second experiment, which examined the potential of compound conditioning within the cross-modal learning procedures, replicated the cross-modal results of the first experiment without finding evidence for compound conditioning. These results are interpreted in terms of specific and nonspecific transfer effects during avoidance learning.
\end{abstract}

In cross-modality learning, if an organism is taught to solve a problem using cues in one sensory modality, then, if some transfer of learning has crossed sensory boundaries, more rapid learning should subsequently occur with a similar problem using cues presented in a different modality. At one time it was thought that humans were capable of making such transfers through verbal mediation, but that nonhuman species were either incapable or only minimally capable of inter- or cross-modality transfer (Ettlinger, 1967; von Wright, 1970). Although much of the subsequent research with nonhuman species has reported negative or, at best, equivocal results with the cross-modal transfer of learning, a growing amount of evidence suggesting cross-modal transfer of discrimination learning has been reported for a number of species, including monkeys (Wilson \& Shaffer, 1963), rabbits (Kehoe \& Holt, 1984; Yehle \& Ward, 1969), dogs (Seraganian \& Popova, 1976), and rats (Meck \& Church, 1982a; Over \& Mackintosh, 1969; Roberts, 1982; Yeterian, 1977). The growth of research literature in this area suggests that nonhuman species are capable of more cross-modal integration during learning than was previously suspected.

Although cross-modal transfer of discrimination learning has been demonstrated for rats, previous attempts to demonstrate cross-modal transfer of avoidance learning in rodents either have been unsuccessful (Gruber, 1969) or were accomplished only by first compounding visual and auditory stimuli (Oliverio \& Bovet, 1969). One exception to this was reported by Delay (1983), who found evidence to suggest cross-modal transfer of avoidance learning in rats. However, Bryant (1968) and others have indicated that to adequately demonstrate cross-modal transfer, within-modality and between-modality direct and

\footnotetext{
The author would like to thank Rona Delay for her assistance in preparing the publication figures.

The author's mailing address is: Department of Psychology, Regis College, West 50th and Lowell Blvd., Denver, CO 80221.
}

reversal transfer procedures should be included as part of the experimental paradigm in order to separate the effects of learning sets from specific cross-modality transfer effects. If specific cross-modality transfer is responsible for any changes in rate of learning the second task, then not only should subjects that are exposed to the second modality learn this task faster than subjects without prior experience with the task using cues in another modality, but the subjects trained with the same intensity relationship (direct transfer) in the second modality should learn more rapidly than the subjects in the reversal condition. However, if both cross-modality intensity conditions produce the same rate of learning within the second condition, then one would have to consider the development of a general response set in which the subject attends to changes related to reinforcement rather than specific information about S+ and S- (Bryant, 1968). Although avoidance training with cues in one modality was found by Delay (1983) to facilitate acquisition of the task in a second modality under direct intensity transfer conditions, transfer effects in reversal conditions were not examined. The first experiment was intended to replicate and expand Delay's (1983) study to more adequately examine the potential of cross-modal transfer in avoidance learning by rats.

\section{EXPERIMENT 1}

This study reexamined Delay's (1983) findings and extended the experimental procedures with appropriate control conditions. If cross-modal transfer is to be demonstrated, savings should be shown in the cross-modal reversal procedures but should not equal the transfer effects seen under direct transfer conditions.

\footnotetext{
Method

Subjects. Forty-eight male and 48 female Sprague-Dawleyderived rats, raised in our colony from breeding stock obtained from
} 
Biological Research Labs, served as subjects. All animals were 90-100 days old and were maintained individually in the colony with food and water available ad lib. Colony lighting was on a 12 h-light:12-h-dark cycle (lights on, 7 a.m.). All testing was done in the light portion of the cycle.

Apparatus. The rats were tested in a four-way shuttle apparatus that measured $24 \times 24$ in. The shuttle, painted flat white throughout, was divided into quadrants with 2 -in. partitions. The grid floor and the top of the partitions were electrified with 0.3-0.4-mA scrambled shock (Delay, 1985), which could be delivered to only one quadrant grid without activating any of the other quadrants. Visual background and conditioned stimulus (CS) lighting was produced by white, incandescent lights located 24 in. above the center of the shuttle and covered with translucent Plexiglas. Auditory background and CS stimulation was generated by an SN76477, an integrated circuit (Texas Instruments) that produces pseudorandom white noise, coupled to an amplifying circuit (Delay, Golden, \& Steiner, 1978). The auditory stimuli were delivered to the shuttle through two speakers mounted in opposite shuttle walls. All auditory measurements were made with a Simpson 886 sound level meter set on the A scale (re: $20 \mu \mathrm{N} / \mathrm{m}^{2}$ ).

Procedures. The rats were handled for at least $5 \mathrm{~min}$ each day for at least 3 consecutive days before initial training. Prior to each session, the subject was allowed to explore the shuttle apparatus for $10 \mathrm{~min}$ while under 70-dB white noise and 160-lx ambient light. For each trial, the CS was initiated 4 sec before shock onset. The animal could escape or avoid the shock and terminate the CS by entering any other quadrant after CS onset. Intertrial intervals, which were randomly determined prior to the experiment to prevent time from becoming a CS (Meck \& Church, 1982b), ranged between 20 and $40 \mathrm{sec}($ mean $=30 \mathrm{sec})$. After each test session, the shuttle apparatus was cleaned and dried.
During the first training session, half of the animals were trained with a visual $C S$ and the rest of the animals were trained with an auditory CS. Of the animals receiving visual training, half were trained with the background light intensity set at $50 \mathrm{~lx}$ and with a CS of $270 \mathrm{~lx}$. The other half were trained in opposite light conditions, that is, with the background lighting set at $270 \mathrm{~lx}$ and with a CS of $50 \mathrm{~lx}$. Background noise was set at $70 \mathrm{~dB}$ whenever a light CS was used. Similarly, of the animals initially receiving auditory training, half began with $60-\mathrm{dB}$ background noise and a CS of $80 \mathrm{~dB}$ and the rest were trained with the opposite auditory conditions. Ambient light was set at $160 \mathrm{~lx}$ when noise was a CS. Three days later, a second session was conducted in which each of the four groups was again subdivided such that an equal number of animals were randomly assigned to one of four training groups. For one group, the same-modality direct (SMD) group, training in the second session employed the same modality and intensity conditions that were used in the original training session. For example, the training of one group was conducted with the high-intensity light $\mathrm{CS}$ in both sessions. The second group, the same-modality reversal (SMR) group, was trained with the same-modality $C S$ in the second session but with CS intensity reversed from that used in original training. For instance, if original training was with the high-intensity light CS, then second-session training used the low-intensity light CS. The third training group was the cross-modal direct (CMD) group, for which training in the second session used the opposite sensory modality but with CS intensity the same as that in original training. For instance, if the original training used the high-intensity light CS, then the second training session used the high-intensity noise CS. The fourth training group was the cross-modal reversal (CMR) group. In the second session, the sensory modality and the CS intensity conditions were the opposite of those used in the original training session. For example, if the original training was with the

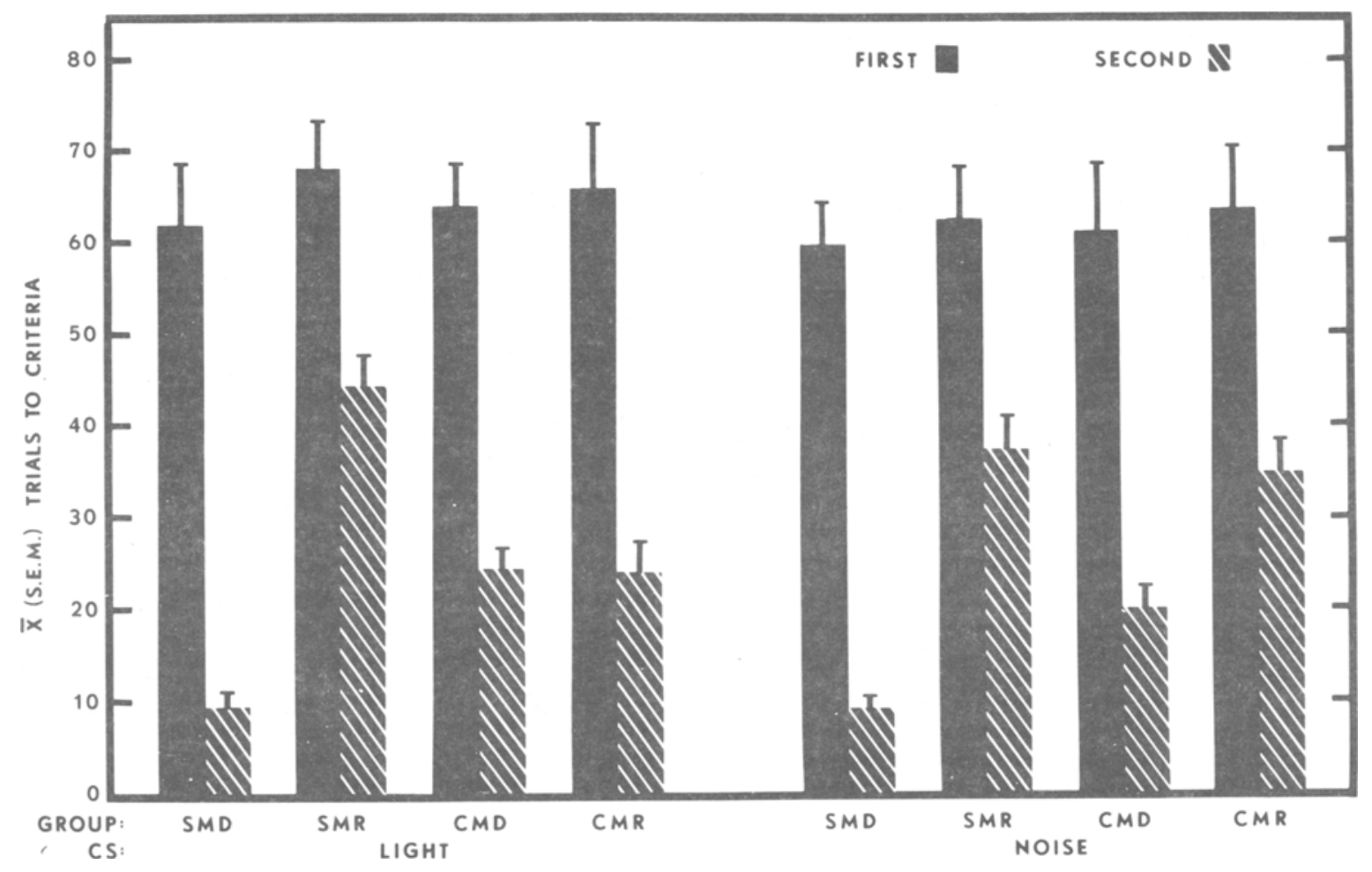

Figure 1. Mean ( $\pm S E M$ ) responses to criteria minus the criterion trials for each group trained under light and noise CSs during the first and second sessions of Experiment 1. 
high-intensity light $\mathrm{CS}$, then the second training session used the low-intensity noise CS. In this manner, sensory modality and intensity were counterbalanced for each training condition. In addition, an equal number of males and females were trained in each condition. All animals were trained to a criterion of 9 avoidance responses in 10 consecutive trials in both training sessions.

\section{Results}

Before the data were analyzed, the 10 criterion trials for each session were subtracted from each animal's data. With an analysis of variance for mixed designs, the data were then examined for effects of the original CS sensory modality (2) and intensity (2), sex (2), training groups (4), and sessions (2). The analysis of variance applied to the raw scores indicated that females acquired the task in significantly fewer trials than males $[F(1,64)=9.37$, $p<.005$ ]. It also revealed significant main effects for training groups $[F(3,64)=7.74, p<.001]$ and sessions $[F(1,64)=260.0, p<.001]$ as well as a significant interaction between these factors $[F(3,64)=5.86$, $p<.005]$. To break the interaction down, an analysis of variance was performed on the data for each training session (Figure 1). The analysis of the first-session data showed only a sex main effect $[F(1,64)=5.07, p<.01]$. However, the analysis of the second-session data indicated significant main effects for sex $[F(1,64)=8.77$, $p<.005]$ and training groups $[F(3,64)=38.17$, $p<.001]$ and a significant interaction between the original sensory modality and training groups $[F(3,64)=3.23$, $p<.05]$. Performance of all groups was better during the second session. Post hoc comparisons (Newman-
Keuls, $p<.05$ ) between the cells of Session 2 indicated that both SMD groups were significantly better than all other groups, that both CMD groups and the light CMR group were similar to each other but significantly different from the rest, and finally, that both SMR and the noise CMR groups were similar to each other but significantly different from the rest of the groups.

To make comparisons with other cross-modal learning studies, a proportional or savings score was generated for each subject by dividing the number of trials to criterion for the second session by the trials to criterion for the first session, again with the criterion trials subtracted from the raw scores. An analysis of variance of these data indicated a significant training groups effect $[F(3,64)=$ $26.21, p<.001]$ and a significant modality $\times$ intensity $\times$ training groups interaction $[F(3,64)=3.14, p<.025$; Figure 2]. Although all groups exhibited savings during the second session, as indicated by a value less than 1.0 , the amount of savings was not uniform across groups and sensory conditions. In both conditions in which the CS was high, the SMD groups showed the best savings and the SMR groups showed the poorest savings scores. The CMD and CMR groups fell between the other two conditions, but the CMD groups exhibited greater savings than the CMR groups. On the other hand, under the low, light CS condition, the CMR group showed better savings than the CMD group, although both groups again fell between the SMD and SMR conditions. Under the low, noise CS condition, the most striking difference in savings was the improved saving score seen for the SMR condition in comparison with the other SMR groups.

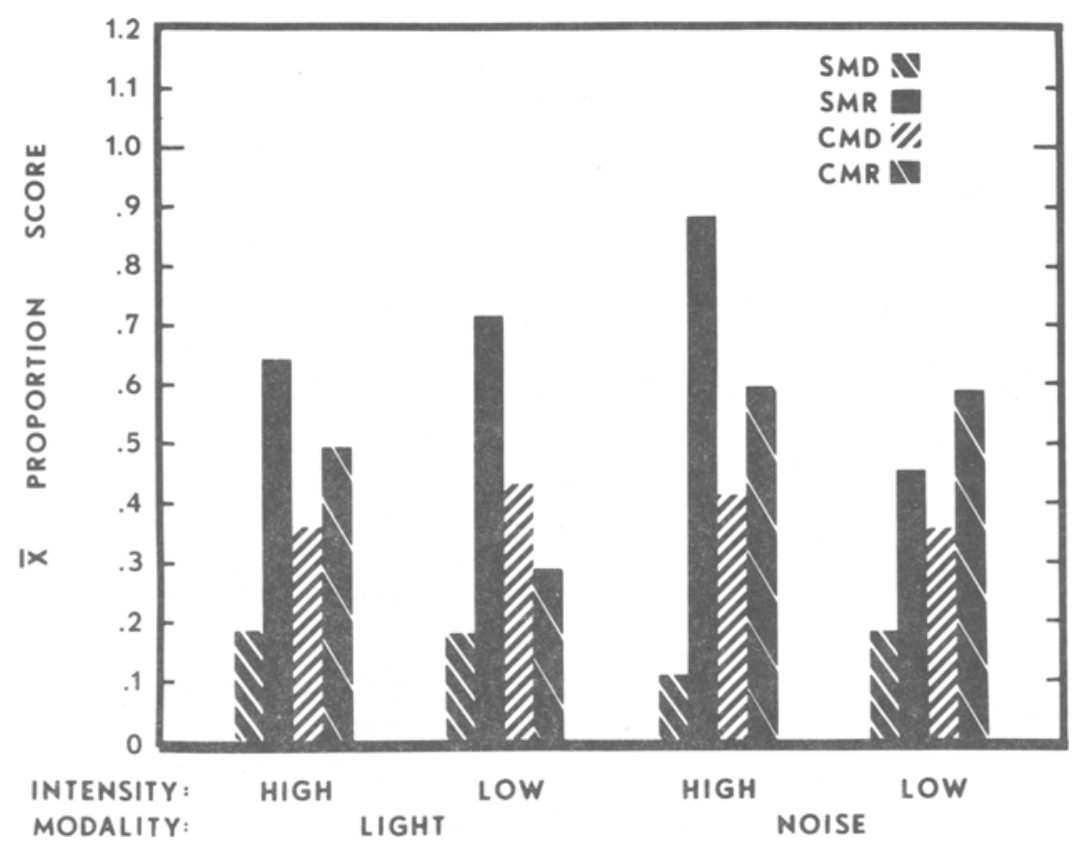

Figure 2. Mean proportion score for each group tested with high- and low-intensity light CSs and with high- and low-intensity noise CSs. The proportional scores were derived by dividing the second session score by the first session score after subtracting the criterion trials for each. This gives an index of savings during the second session of Experiment 1. 


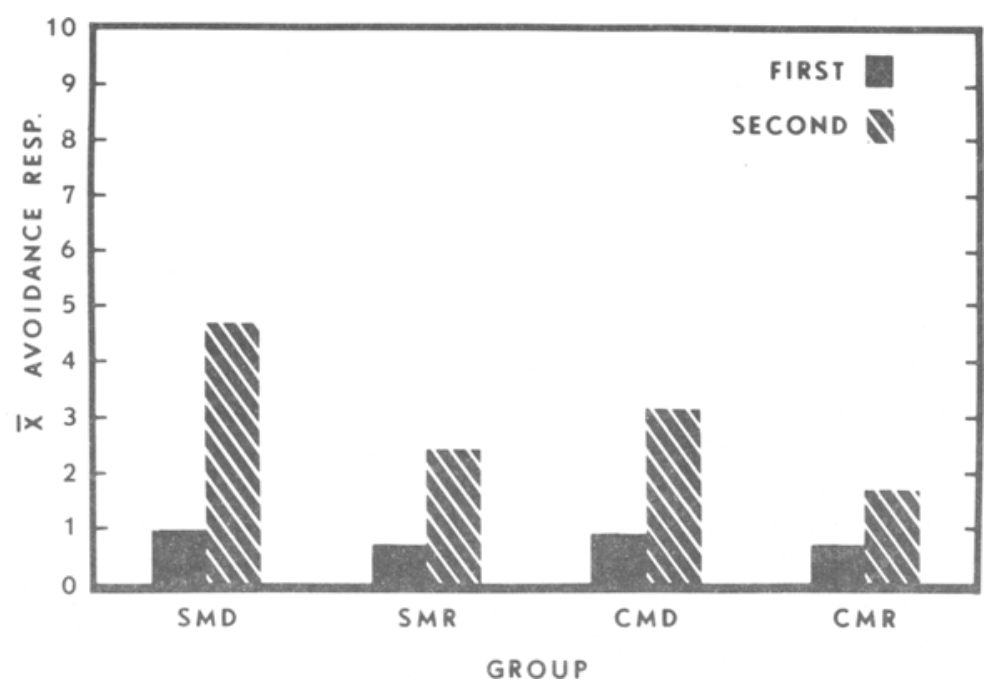

Figure 3. Mean number of avoidance responses during the first 10 trials of the first and second sessions of each training group in Experiment 1.

In addition, the number of avoidance responses made during the first 10 trials of each session was analyzed. The training groups $[F(3,88)=6.39, p<.001]$, sessions $[F(1,88)=84.57, p<.001]$, and training groups $\times$ session $[F(3,88)=5.95, p<.005$; Figure 3$]$ factors were found to be significant. Post hoc testing indicated that the SMD groups made significantly more avoidance responses during the first 10 trials of the second session than did all other groups under all conditions. Also, the second session scores for the SMR and CMD groups differed significantly from those of other conditions but not from each other. However, the number of avoidance responses in the first 10 trials for the CMR group did not change significantly between Sessions 1 and 2 .

The results of Experiment 1, in terms of both raw score data and the proportional data, would appear to suggest that the rat is capable of cross-modal transfer of avoidance learning. However, the basic experimental design was similar to the one used by Over and Mackintosh (1969), one which has been questioned recently by Church and Meck (1983). Essentially, Church and Meck suggested that the CS and the opposite modality background stimulation, maintained at an intermediate intensity value, could form a compound stimulus in each session. If that were true, then the greater the degree of similarity between the characteristics of the positive compound stimulus of the first session and the characteristics of the positive compound stimulus of the second session (e.g., relative intensity values), the better the animal would respond during the second session (Church \& Meck, 1983, pp. 78-79). Rather than involving cross-modal transfer, then, the explanation for the transfer effects of the crossmodal learning groups might be within the framework of a form of compound conditioning. Experiment 2 attempted to explore the potential for compound condition- ing within the cross-modal learning procedures of Experiment 1 .

\section{EXPERIMENT 2}

Probably the most important aspect of Church and Meck's compound conditioning theory involves the idea that the stimulus to be used as a CS for each modality is present in some form in both training sessions, either as the CS for that session or as background stimulation. Compound conditioning, rather than cross-modal transfer, then, may be producing the savings in Session 2. One might be able to present this type of compound conditioning by removing from the background environment the stimulus that is not serving as a CS. By transferring in conditions of differing background environments, Experiment 2 was designed to compare transfer of learning under conditions that could lead to compound conditioning according to the Church and Meck theory. If compound conditioning occurs, then transfer of learning should be better in conditions in which the critical stimuli for compounding are present in both sessions than in conditions in which the stimuli are absent from the background environment when they are not functioning as a CS.

\section{Method}

Subjects. The subjects were 48 male Sprague-Dawley-derived rats, raised in our colony from breeding stock obtained from the University of Colorado Health Science Center. All subjects were 90-100 days of age and were maintained in the colony in the same way as the subjects of Experiment 1 .

Apparatus and Procedures. The shuttle and shock source were the same as those employed in Experiment 1, as were the UCS parameters, the 4-sec CS-UCS temporal relationship, intertrial interval procedures, and the response and learning criteria. When the CS was light, its intensity was $270 \mathrm{~lx}$, with $50 \mathrm{~lx}$ between trials, produced by incandescent lights centered over the shuttle. When 
the CS was white noise, its intensity was $80 \mathrm{~dB}$, with $60 \mathrm{~dB}$ in the intertrial intervals, delivered via the speakers mounted in the shuttle walls.

All animals were given two training sessions 3 days apart. Prior to the first session, 12 animals were assigned randomly to each of four training groups. Two of the groups were used to replicate the procedures for the corresponding groups of Experiment 1. The first group was trained with the high-intensity light CS in the first session with the background white noise set at $70 \mathrm{~dB}$. The second group was trained with the high-intensity white-noise CS, with background incandescent light set at $160 \mathrm{~lx}$. In the second session, 6 rats in each of these groups were given CMD training and the other 6 were given CMR training. During the second session, the stimulus of the noncue modality was set at either $70 \mathrm{~dB}$ for white noise or $160 \mathrm{~lx}$ for light. For all of these animals, then, both stimuli that served as CSs were present at the same time in both sessions, either as the CS or as a background condition.

The subjects in the third and fourth groups also were trained initially with the light and noise CS, respectively, and then each group was subdivided evenly into CMD and CMR training groups for the second session. The principle deviation from previous procedures for these subjects was the absence from the background environment of the stimulus that was not the CS for that session. Specifically, when the CS was the light, the white noise was turned off and a room ventilation fan was the primary source of background auditory stimulation at $48 \pm 2 \mathrm{~dB}$. This fan was not on at any other time during either experiment. When white noise was the CS for these animals, the white incandescent lights were not turned on. Instead, a red incandescent light, mounted $3 \mathrm{ft}$ above one corner of the shuttle apparatus, was turned on to provide $5 \mathrm{~lx}$ of background lighting. These procedures prevented any pairing of the CSs within the two sessions. All animals were allowed to explore the shuttle apparatus for at least $10 \mathrm{~min}$ prior to each session, with the sensory conditions set for the background condition of that session and the CS intensity at either $70 \mathrm{~dB}$ or $160 \mathrm{~lx}$. For Groups 3 and 4 , these procedures also would prevent the occurrence of sensory preconditioning during the habituation period.

\section{Results}

The 10 criterion trials were subtracted from each score before any analyses were performed on these data. The raw data were then examined for potential effects of compounding or pairing of sensory stimuli (2), the sensory modality of the original CS (2), training groups (2), and training sessions (2) with an analysis of variance for mixed designs. The main effect for sessions $[F(1,40)=166.44$, $p<.001]$ and the modality $\times$ sessions interaction $[F(1,40)=4.13, p<.05]$ were significant. Post hoc analysis of this interaction (Figure 4, left panel) indicated that in the first session the rats learned faster with the noise CS than with the light CS and that second-session performance was significantly better for all rats, although CS modality no longer had a significant effect. The analysis of variance also revealed a significant sessions $\times$ groups interaction $[F(1,40)=9.07, p<.005]$. A NewmanKeuls test on the interaction (Figure 4, right panel) indicated that although, in the first session, performance scores of the CMR and the CMD groups were similar to each other, in the second session the performance of the CMR groups improved significantly and the performance of the CMD groups was significantly better in this session than in all other conditions $(p<.05)$. Analyses were performed on the raw data from each training session, but they did not reveal any additional information. Moreover, an analysis of proportional scores revealed that although the CS modality trended toward significant $(p<.06)$, only the group factor was significant $[F(1,40)$ $=51.83, p<.001]$, with the CMD groups showing more savings than the CMR groups (Figure 5). Although the potential for compound conditioning was examined

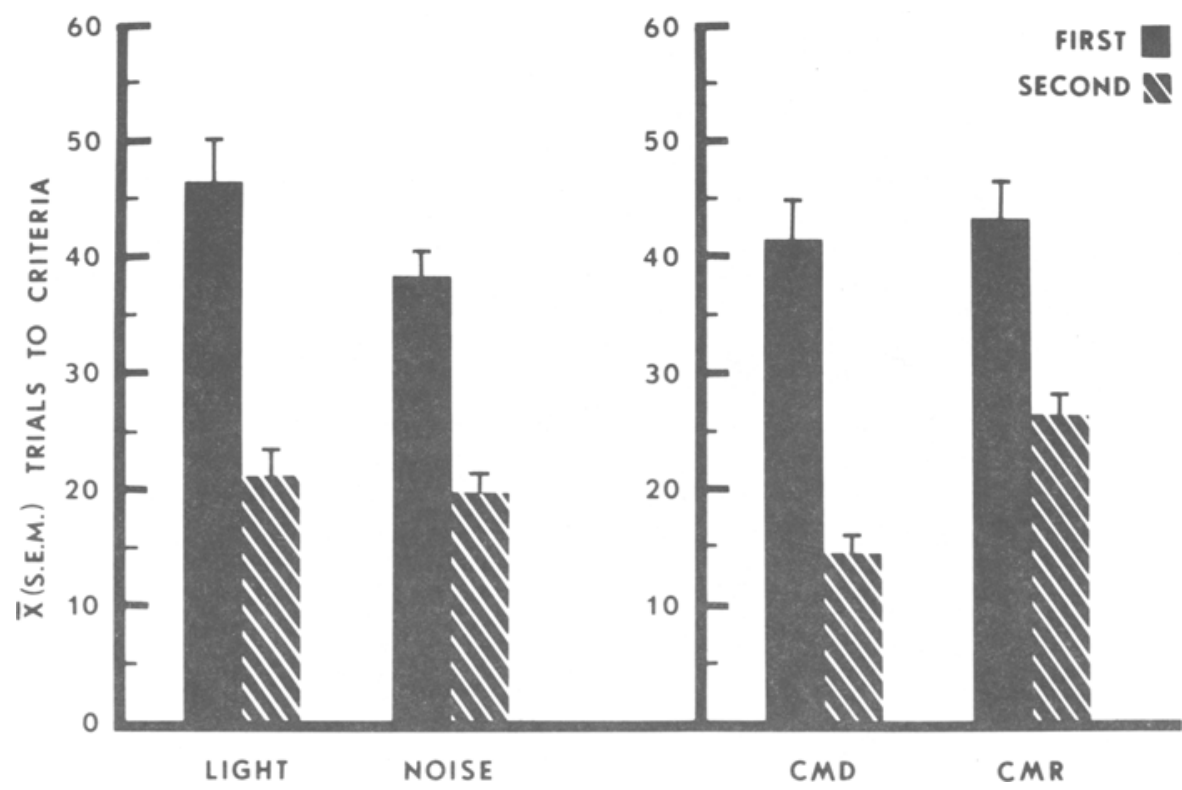

Figure 4. The left panel illustrates the mean $( \pm S E M)$ number of avoidance responses to criteria (minus criterion trials) in each session for training with the light and the noise CSs in Experiment 2. The right panel shows the mean $( \pm S E M)$ number of avoidance responses (minus criterion trials) of the cross-modal direct and cross-modal reversal training groups in each session of Experiment 2. 


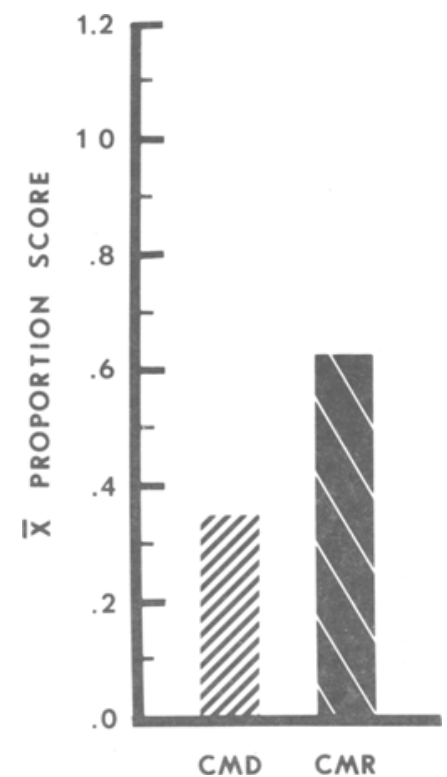

Figure 5. Mean proportional scores showing savings for crossmodal direct and reversal groups in the second session of Experiment 2 .

by each analysis with the pairing factor, at no time did this factor or any of its interactions come close to having a significant effect on trials to criteria or on the amount of savings.

The mean number of avoidance responses for all animals in the first 10 trials of the first session was 0.90 . Analysis of the avoidance responses of the first 10 trials of the second session indicated significant modality $[F(1,40)=9.80, p<.005]$ and groups $[F(1,40)=$ $12.80, p<.001]$ main effects and a modality $\times$ groups interaction $[F(1,40)=5.00, p<.05]$. The CMD group originally trained with the light CS and tested with the noise CS averaged 3.17 avoidance responses in the second session, whereas all other groups averaged 1.17 avoidance responses or less. Again, the pairing factor did not have a significant effect in the second session.

Finally, the acquisition data for Groups 1 and 2 of Experiment 2 were compared with the data for the groups that received identical training in Experiment 1. The raw data, analyzed for the effects of experiments (2), CS modality (2), training groups (2), and sessions (2), indicated significant effects for experiments $[F(1,40)=5.05$, $p<.05]$ and sessions $[F(1,40)=110.14, p<.001]$ and an experiment $\times$ sessions interaction $[F(1,40)=4.48$, $p<.05$ ]. These effects were seen primarily as a smaller number of trials to criteria for the subjects of the second experiment, especially in the first session. A similar analysis of the proportional data found only a training groups effect $[F(1,40)=21.60, p<.001]$, indicating that the CMD groups showed more savings than the CMR groups.

\section{DISCUSSION}

Several studies, using discrimination tasks with foodreinforced barpress-response procedures, have shown that rats are capable of cross-modal learning. For example, rats have been reported to exhibit cross-modal direct and reversal transfer in discrimination tasks with specific visual and auditory cues (Meck \& Church, 1982a; Yeterian, 1977), including ambient light and white noise (Over \& Mackintosh, 1969). On the other hand, neither Gruber (1969) nor Oliverio and Bovet (1969) were able to demonstrate cross-modal transfer of avoidance learning in rodents. However, when one applies Bryant's (1968) criteria for establishing cross-modal learning to the data reported in this study, whether using the raw data or the proportional or savings score data, it would appear that rats are, indeed, also capable of cross-modality transfer in avoidance learning. Church and Meck (1983) have pointed out that studies presuming to demonstrate cross-modal learning have often had common elements of the discriminative stimuli present in the training and testing sessions. Potentially, this situation might allow a rather complex form of compound conditioning to occur that would control behavioral responding in the test session rather than be the result of any form of cross-modal transfer. Furthermore, these authors felt that the selection of intermediate intensity values for the background stimulus during the training session might lead to a bias toward or away from the positive compound stimulus of the test session, depending upon whether the intensity of the second session compound stimulus was the same as or the reverse of the training stimulus. Experiment 2, specifically addressing these possibilities, found that, for both the CMD and the CMR groups, the rate of acquisition and the amount of transfer was not affected by the presence or absence of the opposite-modality CS as part of the background environment. Since the background stimulus in these experiments was unchanging throughout the session, the subject's attention would not be drawn toward it when the UCS was present. Furthermore, since the background stimulus was present during the $\mathrm{CS}$ as well as during the period between each CS, it did not contain any reliable, or novel information concerning the predictability of the UCS, information thought to be needed by each stimulus before compound conditioning can occur (Kamin, 1969; Pearce \& Hall, 1980). However, the results of the present study may be limited to situations involving aversive conditioning or to test conditions in which the CS intensity is unchanging during its presentation. Further study of Church and Meck's (1983) theory is required in cross-modality studies in which, for example, the CS is a temporal pattern that requires a pulsed stimulus.

Two other factors also appeared to affect performance in these experiments. First, in Experiment 1, females learned the task faster in both sessions than males. When sex differences have been reported for active avoidance tasks, females typically have been found to acquire the response faster than males (Beatty, 1977; Denti \& Epstein, 1972; Van Oyen, Walg, \& Van de Poll, 1981). In this study, however, sex differences disappeared when proportional scores were used, which indicated that both sexes exhibited similar savings during the second session. 
It should also be pointed out that the number of trials to criterion for the rats in Experiment 1 was greater than for the animals in Experiment 2, especially in the first session. These differences may have been due to strain differences, because the colony breeding stock was changed during the interval between the experiments. Strain differences have often been noted in active avoidance learning (Bammer, 1983; Barrett, Leith, \& Ray, 1973; Caul \& Barrett, 1973). Nevertheless, the analysis of the proportional data for each experiment indicated that the amount of transfer for the corresponding training groups was similar for both strains. Thus, it does not appear that strain or sex of the subject limit the generality of cross-modal learning seen in these experiments.

One might then ask whether the cross-modal avoidance learning observed in this study was due to nonspecific transfer, specific transfer, or both. It would seem reasonable that at least some of the transfer effects observed in the cross-modal learning groups were due to nonspecific transfer, since identical motor response, test equipment, and test paradigm, with the exception of the CS and ambient background stimulation, were used in both sessions. Ettlinger (1967) has referred to this form of cross-modal transfer as nonspecific supramodal transfer, which might be viewed as a learning-to-learn phenomenon, and would manifest itself, according to Bryant (1968), by equivalent transfer effects in both direct and reversal cross-modal training conditions. Another form of nonspecific transfer might be the result of what Gibson (1969) described as amodal perception, a term that she applied to sensory information that was a relational attribute of stimulation not specific to a modality, such as intensity or temporal patterns. Implicit within this theoretical framework is the notion that the more of these properties common to the training and the test stimuli, the better the cross-modal transfer, regardless of the order of modalities tested. Insofar as intensity was one of the stimulus characteristics manipulated in these experiments, amodal perception could have contributed, at least in part, to the transfer effects seen in these experiments. However, the differential modality and intensity effects seen in the cross-modal learning groups in Experiment 1 suggest that sensoryspecific cross-modal transfer may also have been operating. For example, although the savings scores seen in most of the CMD groups were better than those seen in the CMR groups, this was not a universal effect. The lowintensity light CMR group performed significantly better in the second session than did the corresponding direct transfer group, results that replicated the performance relationship of the identical groups in Over and Mackintosh's (1969) study. These results would suggest that the specific characteristics of the conditioned stimulus differentially affected the magnitude of the transfer effect. In fact, changing the sensory modality had a much greater positive transfer effect than was seen in the SMR groups, groups trained in the second session with the same modality but opposite CS intensity. Similarly, Yeterian (1977) also has found evidence for specific modality and intensity cross-modal transfer effects for discrimination learning in rats, effects that could be disrupted by lesions in either the auditory cortex or the visual cortex of the rat. Furthermore, nonspecific transfer effects would be expected to increase the number of avoidance responses made during the first 10 avoidance trials of the second session. Although this was indeed seen, it was not a strong or general effect in either experiment. For instance, in the second session of Experiment 1, the SMR and the CMD groups showed an increase of only one and a half to a little over two additional avoidance responses over the first session, and the CMR groups did not show a significant change, thus suggesting a differential sensory effect. Furthermore, only one group in Experiment 2, the light CMD group, showed a significant increase in avoidance responding during these trials. These findings are also similar to previous reports of minimal or no immediate transfer effects early in the cross-modal test session (Delay, 1983; Kehoe \& Holt, 1984). Thus, taken as a whole, these data would suggest that, at least for avoidance learning in rats, cross-modal transfer may result from an interaction of nonspecific and sensory-specific transfer processes, all contributing to the positive transfer of learning seen in the second session.

Finally, the finding that most of the SMR groups in Experiment 1 exhibited the poorest transfer effects among all the groups was unexpected, since only the intensity dimension was altered from one session to the next. No consistent behavioral pattern could be discerned from the raw data that would help to explain these differences, nor were there any detectable changes in spontaneous activity between trials. Nonsystematic observations suggested that after the first few trials in the second session, the SMR animals exhibited more freezing responses than did other animals. This plus the performance data suggest that reversing the CS within the same modality also may have produced negative transfer associated with stimuli already conditioned to an aversive UCS, but with the opposite response contingency. This effect, however, may not generalize as much, if at all, when the sensory modality is changed as well. Changing sensory modalities may reduce the stimulus control exerted by the original learning condition and increase the potential for more generalized, cross-modal transfer processes related to intensity discriminations and other task characteristics not explicitly part of the original stimulus modality and response relationship. Overall, it would seem that the negative transfer effects in the SMR groups were relatively weak as compared with the positive transfer effects present, since all of the SMR groups showed savings during the second session. Clearly, further research is needed to elucidate the nature of these phenomena.

\section{REFERENCES}

BAMMER, G. (1983). The Australian high and low avoidance rat strains: Differential effects of ethanol and a-methyl-p-tyrosine. Behavioural Brain Research, 8, 317-333.

BARRETT, R. J., LeITH, N. J., \& RAY, O. S. (1973). A behavioral and 
pharmacological analysis of variables mediating active-avoidance behavior in rats. Journal of Comparative \& Physiological Psychology, 82, 489-500.

BEATTY, W. W. (1977). Sex differences in DRL and active avoidance behaviors in the rat depend upon the day-night cycle. Bulletin of the Psychonomic Society, 10, 95-97.

BRYANT, P. E. (1968). Comments on the design of developmental studies of cross-modal matching and cross-modal transfer. Cortex, 4, 127-137.

CAul, W. F., \& BarretT, R. J. (1973). Shuttle-box versus Y-maze avoidance: Value of multiple response measures in interpreting activeavoidance performance of rats. Journal of Comparative \& Physiological Psychology, 84, 572-578.

ChURCh, R. M., \& MECK, W. H. (1983). Acquisition and cross-modal transfer of classification rules for temporal intervals. In M. L. Commons, R. J. Herrnstein, \& A. R. Wagner (Eds.), Quantitative analyses of behavior: Discrimination processes (Vol. 4, pp. 75-97). Cambridge, MA: Ballinger.

DELAY, E. R. (1983). Cross-modal transfer of avoidance learning in rats. Perceptual \& Motor Skills, 57, 185-186.

DELAY, E. R. (1985). A simple and inexpensive computer-compatible shock circuit. Behavior Research Methods, Instruments, \& Computers, 17, 114-115.

Delay, E. R., Golden, A. J., \& Steiner, N. O. (1978). A compact IC tone generator. Physiology \& Behavior, 21, 133-134.

Denti, A., \& Epstein, A. (1972). Sex differences in the acquisition of two kinds of avoidance behavior in rats. Physiology \& Behavior, 8, 611-615.

EttLinger, G. (1967). Analysis of cross-modal effects and their relationship to language. In C. H. Millikan \& F. L. Darley (Eds.), Brain mechanisms underlying speech and language (pp. 53-60). New York: Grune \& Stratton.

GiBSON, E. J. (1969). Principles of perceptual learning and development (pp. 215-231). New York: Appleton-Century-Crofts.

GrUBER, R. P. (1969). Failure of visual generalization following auditory avoidance training. Psychonomic Science, 17, 269-270.

KAMIN, L. J. (1969). Predictability, surprise, attention, and conditioning. In B. A. Campbell \& R. M. Church (Eds.), Punishment and aversive behavior (pp. 279-296). New York: Appleton-Century-Crofts.
KeHOE, E. J., \& Holt, P. E. (1984). Transfer across CS-US intervals and sensory modalities in classical conditioning of the rabbit. Animal Learning \& Behavior, 12, 122-128.

MeCK, W. H., \& CHURCH, R. M. (1982a). Abstraction of temporal attributes. Journal of Experimental Psychology: Animal Behavior Processes, 8, 226-243.

MeCK, W. H., \& CHURCH, R. M. (1982b). Discrimination of intertrial intervals in cross-modal transfer of duration. Bulletin of the Psychonomic Society, 19, 234-236.

Oliverio, A., \& Bovet, D. (1969). Transfer of avoidance responding between visual and auditory stimuli presented in different temporal patterns. Communications in Behavioral Biology, 3, 61-68.

OVER, R., \& MAckintosh, N. J. (1969). Cross-modal transfer of intensity discrimination by rats. Nature, 224, 918-919.

Pearce, J. M., \& Hall, G. (1980). A model for Pavlovian learning: Variations in the effectiveness of conditioned but not of unconditioned stimuli. Psychological Review, 87, 532-552.

RoBERTS, S. (1982). Cross-modal use of an internal clock. Journal of Experimental Psychology: Animal Behavior Processes, 8, 2-22.

Seraganian, P., \& Popova, Y. I. (1976). Cross modal transfer of a conditional flexion response in dogs. Pavlovian Journal of Biological Sciences, 11, 162-174.

Van Oyen, H. G., Walg, H., \& Van de Poll, N. E. (1981). Discriminated lever press avoidance conditioning in male and female rats. Physiology \& Behavior, 26, 313-317.

VON WRIGHT, J. M. (1970). Cross-modal transfer and sensory equivalence-a review. Scandinavian Journal of Psychology, 11, 21-30.

Wilson, W. A., \& Shaffer, O. C. (1963). Intermodality transfer of specific discriminations in the monkey. Nature, 197, 107.

YeHLE, A. L., \& WARD, J. P. (1969). Cross-modal transfer of a specific discrimination in the rabbit. Psychonomic Science, 16, 269-270.

Yeterian, E. H. (1977). The effects of visual or auditory cortical lesions on specific cross-modal transfer in the rat. Neuropsychologia, $15,517-527$.

(Manuscript received January 13, 1986; revison accepted for publication July 7,1986 .) 